\title{
Experimental Investigation of the Performance of Vertical Handover Algorithms between WiFi and UMTS Networks
}

\author{
Stefano Busanelli ${ }^{1}$, Marco Martalò ${ }^{1}$, Gianluigi Ferrari ${ }^{1}$, \\ Giovanni Spigoni ${ }^{1}$, and Nicola Iotti ${ }^{2}$ \\ 1 WASN Laboratory, Department of Information Engineering, University of Parma, Italy \\ busanelliatlc.unipr.it \\ 2 Guglielmo Srl, Reggio Emilia, Italy \\ nicola.iotti@guglielmo.biz
}

\begin{abstract}
In this paper, we analyze the performance of vertical handover (VHO) algorithms for seamless mobility between WiFi and UMTS networks. We focus on a no-coupling scenario, characterized by the lack of any form of cooperation between the involved players (users and network operators). In this context, we first propose a low-complexity Received Signal Strength Indicator (RSSI)based algorithm, and then an improved hybrid RSSI/goodput version. We present experimental results based on the implementation of a real testbed with commercial WiFi (Guglielmo) and UMTS (Telecom Italia) deployed networks. Despite the relatively long handover times experienced in our testbed, the proposed RSSI-based VHO algorithm guarantees an effective goodput increase at the MTs. Moreover, this algorithm mitigates the ping-pong phenomenon.
\end{abstract}

\section{Introduction}

In order to be Always Best Connected (ABC) [7], the user should be able of taking advantage of the best available access network at any point in time, choosing among the large array of solutions offered by the market, including the various generations of cellular networks (e.g., GSM/GPRS, UMTS, and LTE) [5], metropolitan area networks (e.g., IEEE 802.16 [10] and HiperLAN [4]), wireless local area networks (e.g., IEEE $802.11 \mathrm{a} / \mathrm{b} / \mathrm{g} / \mathrm{n}[8]$ ), and also personal area networks (e.g., Bluetooth [14]). To this end, it is necessary to make the transition of a mobile terminal (MT) between access points belonging to heterogeneous networks possible. A mechanism used to perform this task, possibly in a transparent way from upper-layer applications and "painlessly" from the user perspective, is commonly referred to as Vertical HandOver (VHO). A VHO procedure is composed by three main phases: initiation, decision, and execution [13]. The most relevant international standardization effort regarding VHO, namely the IEEE 802.21 standard [9], refers only to the first two phases (initiation and decision) that are relatively technology-independent, but it deliberately ignores the execution phase. The latter is considered by other standardization bodies, like the 3GPP consortium [5] or the Internet Engineering Task Force (IETF) [11].

\footnotetext{
${ }^{1}$ The access points are also referred as "points of attachment" by some authors (i.e., [1]16]).
} 
In this work, we only focus on a no-coupling scenario, i.e., a scenario without any form of cooperation between the involved players (users and network operators) [13]. In the considered scenario, we adopt a Mobile terminal-Controlled HandOver (MCHO) approach, characterized by the fact that the MT is the only active player in the VHO process [1]. The algorithms presented here are low-complexity extensions of the hybrid Received Signal Strength Indicator (RSSI)/goodput VHO algorithm, between UMTS and WiFi networks, originally presented in [12]. In particular, the latter algorithm is based on the estimation of both the received power and the instantaneous goodput available at each network interface. However, obtaining an accurate goodput estimate is, from a practical viewpoint, very challenging. Therefore, we modify the original VHO algorithm of [12] to derive a pair of simpler, yet with good performance, VHO algorithms suitable to a real-world scenario. The two low-complexity algorithms are described, respectively, in Section 2 and Section 3 , while their performance, evaluated experimentally, is presented and discussed in Section 4. Finally, concluding remarks are given in Section 5 .

In the following, the subscripts $U$ and $\mathrm{W}$ will indicate, respectively, the UMTS and WiFi networks. For the purpose of notational simplicity, the symbol x will be used to indicate a generic interface ( $\mathrm{U}$ or $\mathrm{W}$ ). Moreover, the pair of terms WiFi and IEEE 802.11 and the pair of terms UMTS and $3 \mathrm{G}$ will be used interchangeably.

\section{A Low-Complexity RSSI-Based VHO Algorithm}

The first simplified novel VHO algorithm is derived from the algorithm presented in [12] by applying the following modifications.

- The goodput is no longer considered to make a handover decision.

- The VHO algorithm is asymmetric, as it assigns an implicit preference to the IEEE 802.11 network. In fact, WiFi connectivity is currently less expensive (at least in Italy) than $3 \mathrm{G}$ connectivity. Moreover, our experimental results have shown that the bandwidth offered by IEEE 802.11 networks is typically larger than that offered by $3 \mathrm{G}$ networks.

- The received power is replaced with the RSSI, as the latter can be measured more easily from the received packets.

- The waiting times between consecutive operations are removed, due to their inefficiency.

We now describe the operations of the novel VHO algorithm, with reference to the dataflow shown in Fig. 1 (a). The algorithm is entirely based on the RSSI measurements. Note that in both WiFi and UMTS cases the instantaneous RSSI values are considered. In the WiFi case, the filtered values of the RSSI (denoted as RSSI ${ }_{\mathrm{W}}^{\mathrm{ESA}}$ ) are also considered to avoid instantaneous RSSI peaks in the WiFi network.

The instantaneous RSSI value $\operatorname{RSSI}_{x}$ (of any interface) is compared with two thresholds, denoted as $\mathrm{TH}_{\mathrm{x}}^{\mathrm{U}}$ and $\mathrm{TH}_{\mathrm{x}}^{\mathrm{L}}$. The lower threshold $\mathrm{TH}_{\mathrm{x}}^{\mathrm{L}}$ is used to determine when the RSSI is not sufficient to guarantee a stable connectivity: therefore, it is slightly higher than the corresponding interface sensitivity. Clearly, when $\mathrm{RSSI}_{\mathrm{x}}<\mathrm{TH}_{\mathrm{x}}^{\mathrm{L}}$ the connection on the interface $\mathrm{x}$ is turned down. On the other hand, the upper threshold 


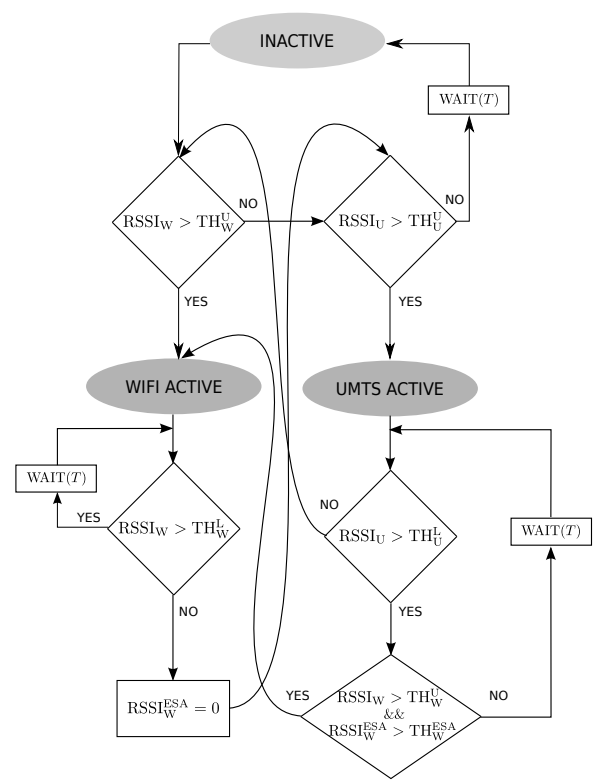

(a)

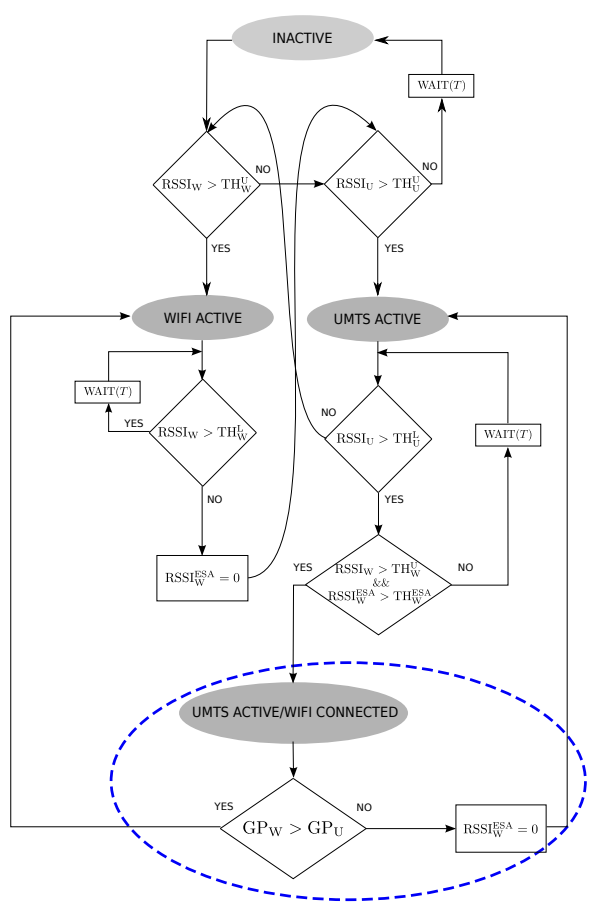

(b)

Fig. 1. (a) Dataflow of the RSSI-based VHO algorithm. (b) Dataflow of the hybrid RSSI/goodputbased VHO algorithm.

$\mathrm{TH}_{\mathrm{x}}^{\mathrm{U}}$ is used to determine if the measured RSSI is sufficient to establish a stable connection. To this end, we assume that $\mathrm{TH}_{\mathrm{x}}^{\mathrm{U}}>\mathrm{TH}_{\mathrm{x}}^{\mathrm{L}}$. The use of two thresholds (per network interface) is the first countermeasure against the ping-pong effect and was historically introduced in the context of cellular networks for managing horizontal handovers.

The average WiFi RSSI values are obtained with the following Exponential Smoothing Average (ESA) filtering:

$$
\left\{\begin{array}{l}
\operatorname{RSSI}_{\mathrm{W}}^{\mathrm{ESA}}(n)=v_{1} \operatorname{RSSI}_{\mathrm{W}}(n)+v_{2} \operatorname{RSSI}_{\mathrm{W}}^{\mathrm{ESA}}(n-1)+v_{3} \operatorname{RSSI}_{\mathrm{W}}^{\mathrm{ESA}}(n-2) \\
v_{1}, v_{2}, v_{3} \in[0,1] \\
\sum_{i=1}^{3} v_{i}=1
\end{array}\right.
$$

where $\left\{v_{1}, v_{2}, v_{3}\right\}$ are proper weighing coefficients and $n$ is the time epoch. The $\operatorname{RSSI}_{\mathrm{W}}^{\mathrm{ESA}}$ values are compared to another threshold, denoted as $\mathrm{TH}_{\mathrm{W}}^{\mathrm{ESA}}$. Unlike the instantaneous RSSI, used to force quick decisions, the filtered RSSIs are used to make "effective" ABC decisions. Moreover, the use of average measurements is expedient to further mitigate the annoying ping-pong phenomena.

According to Fig. 1 (a), the MT can be in three different self-explanatory states: INACTIVE, WiFi ACTIVE, and UMTS ACTIVE. When in the INACTIVE state, the MT measures, with period $T$ (dimension: [s]), the RSSI level at each network interface. 
As soon as the first (of the two) RSSI level overcomes its upper threshold, the corresponding interface notifies the event to the VHO manager, triggering the execution of the Authentication, Authorization, and Accounting (AAA) procedure to join the selected network. We observe that if both networks are available, the priority is always given to the WiFi network. If the AAA procedure in the selected network x succeeds, the state of the MT switches from INACTIVE to "x ACTIVE."

When in the UMTS ACTIVE state, the MT periodically (with period $T$ ) compares $\mathrm{RSSI}_{U}$ with the lower threshold $\mathrm{TH}_{\mathrm{U}}^{\mathrm{L}}$. If $\mathrm{RSSI}_{\mathrm{U}}<\mathrm{TH}_{\mathrm{U}}^{\mathrm{L}}$, the handover manager immediately starts the authentication of the WiFi network, after verifying that $\mathrm{RSSI}_{\mathrm{W}}>$ $\mathrm{TH}_{\mathrm{U}}^{\mathrm{U}}$. If the latter condition is not satisfied, the VHO manager is forced to turned down the UMTS connection and the MT switches to the INACTIVE state. On the other hand, if $\mathrm{RSSI}_{\mathrm{U}}$ remains higher than the threshold $\mathrm{TH}_{\mathrm{U}}^{\mathrm{L}}$, the manager has the opportunity of "quietly" evaluating the status of the WiFi network, in order to assess the possibility of performing a handover. In particular, the algorithm performs a double check, verifying that $\mathrm{RSSI}_{\mathrm{W}}>\mathrm{TH}_{\mathrm{W}}^{\mathrm{U}}$ and that $\mathrm{RSSI}_{\mathrm{W}}^{\mathrm{ESA}}>\mathrm{TH}_{\mathrm{W}}^{\mathrm{ESA}}$. In the case of success, the VHO manager starts to re-route the user traffic on the IEEE 802.11 interface and begins the log-off procedure on the UMTS network 2

When the MT is in the WiFi ACTIVE state, the behavior of the VHO algorithm is different from that when the MT is the UMTS ACTIVE state. In fact, in this case, until $\mathrm{RSSI}_{\mathrm{W}}$ remains higher than the threshold $\mathrm{TH}_{\mathrm{W}}^{\mathrm{L}}$, the MT is forced to remain in the WiFi ACTIVE state, ignoring the conditions on the UMTS interface. Only when the WiFi connectivity is lost $\left(\mathrm{RSSI}_{\mathrm{W}}<\mathrm{TH}_{\mathrm{W}}^{\mathrm{L}}\right)$, the VHO manager compares the $\mathrm{RSSI}_{\mathrm{U}}$ with the threshold $\mathrm{TH}_{\mathrm{U}}^{\mathrm{U}}$, in order to initiate the AAA procedure in the UMTS networkas already explained, this inner preference for the WiFi network is only motivated by real-life experience. Before the AAA operation is started, the filtered value of the RSSI is set to zero in order to prevent rapid re-connections to the WiFi network 3

\section{A Simplified Hybrid RSSI/Goodput VHO Algorithm}

We now propose another VHO algorithm that builds on the previous one, but makes also use of goodput information. This extension aims at avoiding switching from the UMTS network to the WiFi network when the latter offers a smaller effective bandwidth. Although this extension goes back to the approach proposed in [12], the complexity will be kept lower.

The dataflow of the new hybrid algorithm is shown in Fig.1(b). By comparing this dataflow with the dataflow of the RSSI-based algorithm (Fig. 1 (a)) there is an additional state, the WiFi CONNECTED/UMTS ACTIVE state (highlighted at the bottom), where the MT is authorized in both networks. The presence of this state is expedient to estimate the bandwidths of both networks. The bandwidth is directly estimated by

\footnotetext{
${ }^{2}$ The re-routing of existent connections needed to have seamless connectivity after a handover is a problem not addressed in this work. Therefore, after a handover the pre-existent user connections will be likely interrupted.

${ }^{3}$ Note that the same result can be obtained by inserting a delay with fixed duration $T$. However, our solution allows to continuously check the $\mathrm{RSSI}_{U}$ which cannot be done with the fixed delay.
} 
measuring the time necessary to download a 400 Kbyte file from a remote host (for the ease of simplicity, the file is hosted by a Google server), using the HTTP over TCP protocol. Since the throughout test is performed during a walk, there is a tradeoff between the duration of the download and the estimate accuracy. In fact, the distance covered during a single test is inversely proportional to the effective data rate. In order to limit this effect, we impose a double timeout over the download test: (i) a timeout of $1 \mathrm{~s}$ for the establishment of the HTTP connection; (ii) a timeout of $1 \mathrm{~s}$ for the data reception from the remote server.

Due to the asymmetric nature of the algorithm, the MT can move towards the WiFi CONNECTED/UMTS ACTIVE state only from the UMTS ACTIVE state. In particular, during this transition the MT performs the AAA to the IEEE 802.11 network. Then, the MT remains in the WiFi CONNECTED/UMTS ACTIVE state for all the time needed to estimate the goodputs of both networks. As soon as the new measurements, denoted as $\mathrm{GP}_{\mathrm{W}}$ and $\mathrm{GP}_{\mathrm{U}}$, are available, the $\mathrm{VHO}$ algorithm decides to switch to the WiFi ACTIVE or to come back to the UMTS connected state. In the latter, the MT disconnects from the WiFi network and resets its filtered RSSI, in order to reduce the waste of resources. From a practical point of view, when $\mathrm{RSSI}_{\mathrm{W}}^{\mathrm{ESA}}>\mathrm{TH}_{\mathrm{W}}^{\mathrm{ESA}}$ the goodput is periodically estimated with a variable but small frequency, given by the minimum between the time necessary to complete the AAA procedure and the time necessary to fill again $\mathrm{RSSI}_{\mathrm{W}}^{\mathrm{ESA}}$.

\section{Experimental Results}

\subsection{Experimental Setup}

In order to analyze the behavior of our VHO algorithm in a realistic environment, we perform our test using a notebook running the Windows 7 OS, equipped with a Broadcom IEEE $802.11 \mathrm{~g}$ compliant network interface and integrated by a UMTS USB Huawei dongle, using the UMTS standard. We have implemented the VHO mechanism proposed in [12] and its novel low-complexity modifications (presented in Section 2 and Section 3 ) on top of a so-called Smart Client (SC) software 4 The SC has also to take care of the suitable AAA procedure and, due to the make-before-break approach, has also to manage the routing functionalities of the OS, in order to make non-critical the multi-homed situation that appears after the authentication on the second network interface [3, 15].

The IEEE 802.11 connectivity was offered by a hotspot owned by one of the biggest Italian Wireless Internet Service Provider (WISP), namely Guglielmo S.r.1. [6]. The hotspot is composed by a Browan IEEE 802.11 access point, integrated with a captive portal, while the Authentication Server (AS) is remotely located, as in the standard WISP Roaming (WISPR) configuration [2]. The proprietary AAA procedure foresees two additional message exchanges with respect to the WISPR directives [2], thus increasing the time needed to complete the AAA procedure. The UMTS connectivity was instead offered by the Public Land Mobile Network (PLMN) of Telecom Italia, one of

\footnotetext{
${ }^{4}$ The current implementation of the SC runs on a Windows 7 platform, but also supports the Microsoft Vista OS.
} 
the most important Italian mobile operator. The sequence of messages needed to complete the AAA procedure has been considered as that of a typical $3 \mathrm{G}$ network (3GPP recommendations) [5].

The values of the parameters of the VHO algorithm used in the experiments are: $v_{1}=0.08, v_{2}=0.15, v_{3}=0.77$, and $T=0.5 \mathrm{~s}$. Our experiments were conducted in a building within the Department of Information Engineering of the University of Parma. The nearest UMTS base station is placed roughly at $1 \mathrm{Km}$ from the building, and it offers a 2 Mbit/s downlink (384 Mbit/s uplink) bandwidth, being compliant with the UMTS specifications. We have placed the hotspot in the WASN Lab, at $1 \mathrm{~m}$ above the ground. The hotspot is fed by an optical fiber network with $100 \mathrm{Mbit} / \mathrm{s}$ of symmetric bandwidth, but the hotspot imposes a symmetric limit on the available bandwidth equal to $2 \mathrm{Mbit} / \mathrm{s}$, similar to that provided to typical customers. Despite the identical nominal downlink UMTS bandwidth (2 Mbit/s), the WiFi network has often outperformed, in our tests, the UMTS network. The tests were always performed during working hours, in order to obtain results associated with realistic daylife situations.

The first considered performance metric is the handover time, defined as the duration between initiation and completion of the handover process. The initiation corresponds to the instant when the VHO manager begins the AAA procedure to connect to a given network. The completion corresponds to the instant at which the first data packet routed via the new connection is successfully acknowledged by the remote destination. The other metric of interest is the goodput, which refers to the data rate delivered to the MTs in the network [16].

\subsection{Low-Complexity RSSI-Based VHO Algorithm}

The handover time is automatically measured by the SC. We average over 20 different runs: in each run, the path in the building and the corresponding handover instant have changed. In practice, we have collected the handover time, relative to 88 UMTS $\rightarrow$ $\mathrm{WiFi}$ and $88 \mathrm{WiFi} \rightarrow$ UMTS transitions.

In Fig. 2, the instantaneous values of handover time for each transition is shown. From Fig. 2 (a), it emerges that WiFi and UMTS networks have a very different behavior. In particular, the UMTS network exhibits an almost constant handover time, around its average of $4.13 \mathrm{~s}$. However, there is a relevant number of samples also in the region between $5 \mathrm{~s}$ and $10 \mathrm{~s}$, while the few values above $10 \mathrm{~s}$ can be considered as outliers. On the other hand, the WiFi network is definitively worse than the UMTS network, as the handover time presents a higher average value $(5.43 \mathrm{~s})$ and a much higher standard deviation $(3.30 \mathrm{~s})$. At the same time, one should observe that the minimum value is very small (1.22 s).

For the sake of completeness, we have also estimated, upon time discretization in $0.25 \mathrm{~s}$ bins, the Probability Mass Function (PMF) of the handover time. The obtained PMFs, for the two VHO operations, are shown in Fig. 2 (b). Observing the upper subfigure in Fig. 2(b), it seems that the handover time from the UMTS network to the WiFi network spreads between $1 \mathrm{~s}$ and $10 \mathrm{~s}$. This relatively high variability has several motivations. First of all, in order to save energy, the MT is supposed to logout from a given network once the VHO manager has selected the other network. Sometimes (more often in the WiFi network), the logout fails and the remote authentication server keeps the 

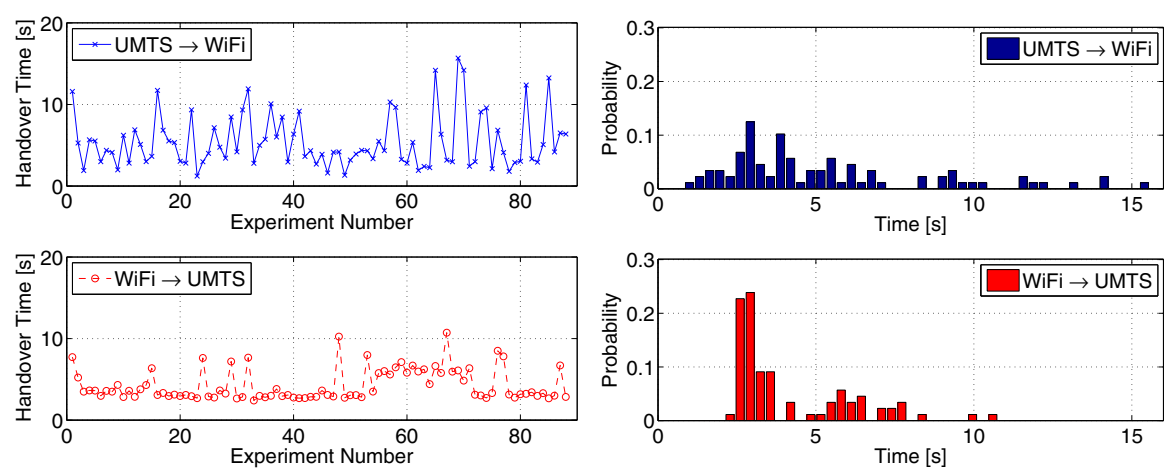

(a)

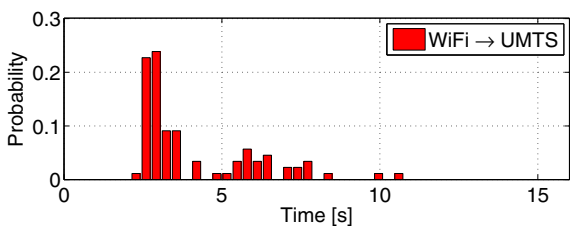

(b)

Fig. 2. (a) Instantaneous handover time of the VHO algorithm presented in Section 2 (b) PMFs of the handover time of the VHO algorithm presented in Section 2 from UMTS to WiFi (upper) and from WiFi to UMTS (lower).

authentication state for a certain timeout (roughly $60 \mathrm{~s}$ ), before automatically logging the user out. In these cases, the UMTS $\rightarrow$ WiFi transitions can experience a short handover time since the MT is de-facto already authenticated to the network. Moreover, while the authentication procedure at MAC layer has, in practice, no impact, the release of an IP address by a DHCP (WiFi network) introduces significant randomness. Finally, when the WiFi signal is received close to furniture, the RSSI experiences large oscillations that can delay the AAA procedure. Conversely, the RSSI of the UMTS network is more stable and the probability of experiencing such large variations is very small.

From the results shown in the lower subfigure in Fig. 2 (b), it can be observed that the handover time from the WiFi network to the UMTS network of the UMTS AAA is generally shorter and more predictable (i.e., its PMF is more concentrated) than that in the opposite direction. However, due to the no-coupling and the lack of any optimization, the handover times are long, also in the case of the UMTS network. This result has somehow to be expected, since the proposed algorithm is designed to be used for slowly mobile MT, e.g., people moving from a place to another.

In order to measure the goodput, we focus on a single walking path, chosen among the experimental data set. The selected path is shown in Fig. 3(a), along with the layout of the environment where the tests were performed. The bold solid lines represent reinforced concrete walls, that are source of a strong signal attenuation. In correspondence to a glass window or a door (where the bold solid lines are interrupted), the signal attenuation is clearly much weaker. The path followed by the user is represented by a dashed line and is delimited by the words "START" and "END." The circles drawn along the path represent the measured available goodput: in particular, the diameter of the circle is proportional to the available goodput. In correspondence to filled circles, data was sent via the IEEE 802.11 interface, while in correspondence to the empty circles the UMTS interface was used. A (filled) diamond denotes the beginning of a VHO procedure, while a triangle indicates when the procedure has been successfully completed. The filled triangles indicate that the VHO procedure has established a WiFi connection, 


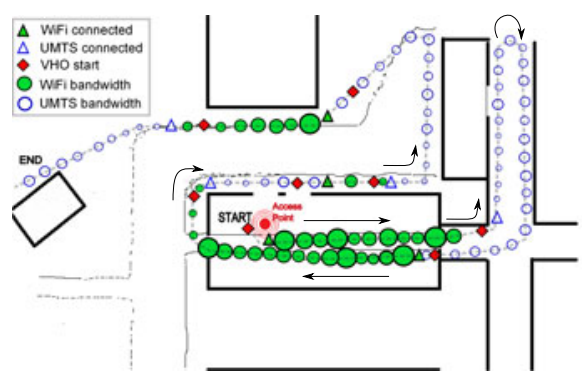

(a)

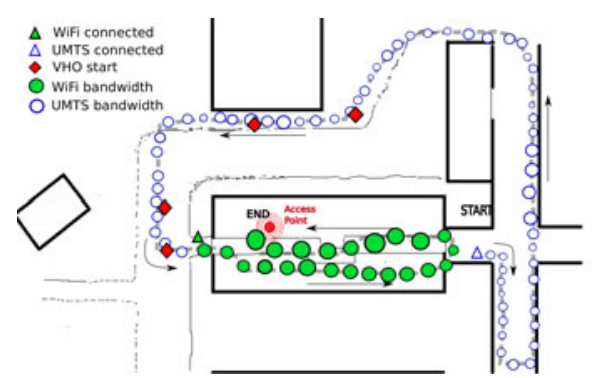

(b)

Fig. 3. (a) Throughput improvement using the RSSI-based algorithm presented in Section 2 (b) Throughput improvement using the hybrid RSSI/goodput-based algorithm presented in Section 3 .

while empty triangles denote the establishment of a UMTS connection. We stress the fact that between diamonds and triangles the MT is still connected with the old network, in order to avoid loss of connectivity before finalizing the VHO. Finally, the distance between the circles is directly proportional to the duration of the bandwidth test and, hence, it is inversely proportional to the available bandwidth.

\subsection{Low-Complexity Hybrid RSSI/Goodput VHO Algorithm}

The performance of the hybrid VHO algorithm has been analyzed considering a slightly different path in the experimental scenario, shown in Fig. 3 (b). In this particular scenario, the assumption of a higher bandwidth of the WiFi network, with respect to that of the UMTS network, is not satisfied. As in Fig. 3 (a), the diamonds in Fig. 3 (b) indicate the beginning of handover procedures. In this case, the handover may fail with a higher probability, due to the additional goodput check. This motivates the presence, in Fig. 3(b), of several diamonds not followed by triangles.

In Fig. 4, the RSSI and goodput relative to the VHO algorithm introduced in Section 3 are shown as functions of time. For the sake of comparison, a direct comparison with the RSSI-based VHO algorithm is also considered. In the upmost subfigure, $\mathrm{RSSI}_{\mathrm{W}}$ is shown together with the corresponding upper and lower thresholds; in the lowest subfigure, the estimated goodputs $\mathrm{GP}_{\mathrm{W}}$ and $\mathrm{GP}_{\mathrm{U}}$ are directly compared; finally, in the middle subfigure the overall goodput guaranteed by the RSSI-based VHO algorithm (denoted as GP) and the hybrid VHO algorithm (denoted ad $\mathrm{GP}^{H}$ ) are directly compared. According to the results in Fig. 4, in the initial phase the MT is disconnected from the WiFi network, because of the too low received power. At a given point of the path, $\mathrm{RSSI}_{\mathrm{W}}$ starts to quickly increase, and then it soon overcomes the threshold $\mathrm{TH}_{\mathrm{W}}^{\mathrm{U}}$. A few seconds later, therefore, the filtered RSSI also goes over its threshold $\left(\mathrm{RSSI}_{\mathrm{W}}^{\mathrm{ESA}}>\mathrm{TH}_{\mathrm{W}}^{\mathrm{ESA}}\right)$. At this moment, the RSSI-based algorithm begins the VHO procedure to the WiFi network, ignoring the fact that the effective goodput available in the WiFi network is lower. On the other hand, in the case of the hybrid algorithm, the MT starts the bandwidth estimation process, after which it decides to keep the UMTS connection because it becomes aware of the higher goodput offered by the UMTS network. In other words, the hybrid VHO algorithm has shown to have better goodput 

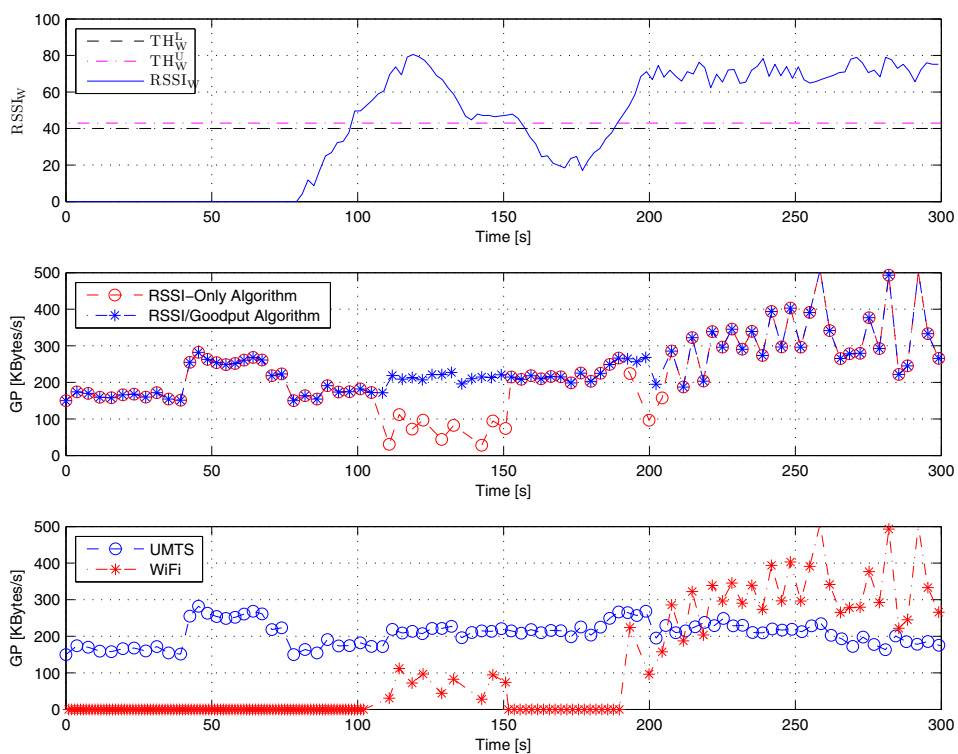

Fig. 4. RSSI and goodput experienced by the MT following a sample path using the hybrid RSSI/goodput VHO algorithm

performance than that of the RSSI-based algorithm, the only differences being slightly higher handover time and complexity. These prices to be paid are due to the presence of a double connectivity situation, which requires to properly configure the OS routing table, in order to perform the bandwidth test on both networks, without penalizing the user.

In terms of handover time, the hybrid VHO algorithm has shown similar performance to the RSSI-based one, the only difference being a longer handover time in the UMTS $\rightarrow$ WiFi transition because of the time needed to perform the bandwidth test. The additional delay is upper bounded by the sum of the two timeouts introduced in Section 3 (HTTP connection and data reception), which is approximately equal to $2 \mathrm{~s}$ in the standard configuration.

\section{Concluding Remarks}

In this paper, we have proposed two simplified novel VHO algorithms (the first one relying on RSSI measures and the second on RSSI and goodput measures) and analyzed their performance experimentally. It has been shown that the VHO procedure in loosely-coupled heterogeneous networks experiences a long handover time, mostly due to the latency induced by the AAA procedures currently under use in IEEE 802.11 and $3 \mathrm{G}$ networks. This problem is exacerbated in the handover from UMTS to WiFi networks. The proposed VHO algorithms, nevertheless, are feasible in the presence of pedestrian mobility. Although an accurate goodput estimate is difficult to obtain in real 
environments, the second proposed $\mathrm{VHO}$ algorithm leverages on rare periodic goodput estimations to guarantee $\mathrm{ABC}$ conditions to the user.

\section{Acknowledgment}

This work was sponsored by Guglielmo Srl. The work of G. Spigoni was also partially supported by a Spinner scholarship. We would like to thank Ing. G. Guerri (Guglielmo Srl) for his continuous support and help. The authors would also like to thank Dr. A. M. Vegni (University of Roma 3) for insightful discussions.

\section{References}

1. Akyildiz, I.F., Mcnair, J., Ho, J.S.M., Uzunalioglu, H., Wang, W.: Mobility management in next-generation wireless systems. Proc. IEEE 87(8), 1347-1384 (1999)

2. Anton, B., Bullock, B., Short, J.: Best current practices for wireless internet service provider (WISP) roaming. Tech. Rep., Wi-Fi Alliance (February 2003)

3. Braden, R.: IETF RFC 1122, Requirements for Internet Hosts - Communication Layers (1989)

4. European Telecommunications Standards Institute: HIPERLAN Type 2 Broadband Radio Access Networks (BRAN); System Overview (February 2000)

5. 3rd Generation Partnership Project: 3GPP, http: //www . 3 gpp. org

6. Guglielmo S.r.l., www .guglielmo.biz

7. Gustafsson, E., Jonsson, A.: Always best connected. IEEE Wireless Commun. Mag. 10(1), 49-55 (2003)

8. Insitute of Electrical and Electronics Engineers: IEEE Std 802.11TM-2007. Part 11: Wireless LAN Medium Access Control (MAC) and Physical Layer (PHY) specifications (2007)

9. Insitute of Electrical and Electronics Engineers: IEEE Std 802.12TM-2008. Part 21: Media Independent Handover Services (2008)

10. Insitute of Electrical and Electronics Engineers: IEEE Std 802.16TM-2009. Part 16: Air Interface for Broadband Wireless Access Systems (2009)

11. Internet Engineering Task Force, http: / / www. ietf . org/

12. Inzerilli, T., Vegni, A.M.: A reactive vertical handover approach for WiFi-UMTS dual-mode terminals. In: Proc. of IEEE International Symposium on Consumer Electronics (ISCE), Algarve, Portogallo, pp. 1-4 (April 2008)

13. Lampropoulos, G., Passas, N., Merakos, L., Kaloxylos, A.: Handover management architectures in integrated WLAN/cellular networks. IEEE Communications Surveys \& Tutorials 7(4), 30-44 (2005)

14. SIG Bluetooth: Bluetooth Specification. Version 4.0 (2010)

15. Yan, X., Sekercioglu, Y.A., Narayanan, S.: A survey of vertical handover decision algorithms in 4G heterogeneous wireless networks. Computer Networks 54(11), 1848-1863 (2010)

16. Wasserman, M., Seite, P.: IETF Draft (Work in progress) - Current Practices for Multiple Interface Hosts (2010) 